\title{
Hacia una nueva Revista, con la ayuda de todos
}

\author{
Towards a new Journal, with the help of all
}

Carlos Andrés Celis Preciado, MD ${ }^{(1)}$; Milena García, MD(2)

Desde su primer número, en 1989, la Revista Colombiana de Neumología ha dado testimonio de la evolución y el desarrollo de la Neumología y la Cirugía del Tórax en el país, convirtiéndose en el espacio de publicación y consulta de la investigación y el pensamiento de neumólogos y cirujanos de tórax, así como de disciplinas afines.

Es hora, no obstante, de que la Revista asimile los cambios que se han dado en los últimos años en cuanto a publicaciones biomédicas colombianas e internacionales (1), e implemente estrategias que le permitan sobrevivir y diferenciarse de las demás revistas de la subespecialidad, lo que implica retos en varios aspectos $(2,3)$.

Uno de ellos es la inclusión de la revista en las bases SciELO, Red de Revistas Científicas de América Latina y el Caribe, España y Portugal (Redalyc), Latinindex, Publindex de COLCIENCIAS y, más adelante, a PubMed. Para lograrlo se planean cambios en los Comités Editorial y Científico, se mantendrá el número mínimo de artículos originales y de revisión, se instaurará formalmente la revisión por pares de los artículos (4) y se verificará el plagio, entre otras acciones.
El sometimiento de los artículos debe ser un proceso cada vez más sencillo y claro para los autores, y los tiempos para revisión y cambios cada vez más cortos, de manera que la información pueda aparecer en línea, incluso antes de ser publicada de forma impresa. Para dinamizarlo se planea que dicho proceso se realice exclusivamente a través de la página de la Revista en una plataforma diseñada para ello; esto permitirá disminuir el tiempo total de la revisión y facilitará una comunicación más cercana y fluida con los autores.

Con relación al contenido de la Revista, se dispondrá de nuevas secciones y se dará más relevancia al contenido interactivo en la red, de modo que los artículos puedan enriquecerse con material exclusivo en línea, como tablas y figuras adicionales o videos, así como respuestas de los lectores en tiempo real, cuya publicación está limitada en el impreso.

Todos estos cambios solo pueden hacerse si se cuenta con un flujo constante de material, de ahí que nuevamente se hace un llamado a todos los neumólogos y cirujanos de tórax del país a que envíen sus investigaciones; la Revista y sus editores tienen el compromiso de ayudarlos en el proceso
(1)Internista, Neumólogo. Unidad de Neumología, Departamento de Medicina Interna, Hospital Universitario San Ignacio. Pontificia Universidad Javeriana. Bogotá, Colombia.

(2)Internista, Departamento de Medicina Interna, Hospital Universitario San Ignacio. Pontificia Universidad Javeriana. Bogotá, Colombia. Correo electrónico: revistacolneumología@gmail.com 
en el menor tiempo posible. También nos dirigimos muy especialmente a los directores de programas de Neumología y Cirugía de Tórax; la Revista es el medio ideal para que sus fellows aprendan la tarea de escribir y publicar y debería considerarse como la primera elección para llevar a feliz término el primer acercamiento a dicha tarea.

Así mismo, invitamos a especialidades afines como Neumología Pediátrica y Terapia/Rehabilitación Respiratorias a que sometan sus investigaciones a la Revista y consolidemos juntos una publicación fuerte en el área de la enfermedad respiratoria.

\section{Bibliografía}

1. Smith R. The trouble with medical journals. J R Soc Med. 2006;99:115-9.

2. Dinh-Xuan AT, Brusasco V. The ERJ in its 21 st. year of age: a smooth transition for an everlasting evolution. Eur Respir J. 2009;33:1-2.

3. Irwin RS, Tedeschi V, Rice J, French CT, Welch SJ. CHEST Launches a new era with a new design. Chest. 2014;146:1-4.

4- Migliori GB, Soriano JB, Brusasco V, Dinh-Xuan AT. ERJ peer reviewers: does this pillar of the Journal's quality need help? Eur Respir J. 2011;38:251-2. 